\title{
Population Pharmacokinetics of Upadacitinib in Healthy Subjects and Subjects with Rheumatoid Arthritis: Analyses of Phase I and II Clinical Trials
}

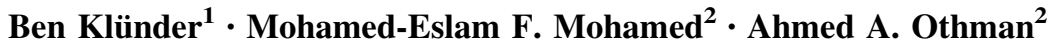

Published online: 26 October 2017

(C) The Author(s) 2017. This article is an open access publication

\begin{abstract}
Background and Objectives Upadacitinib is a janus kinase (JAK) 1 inhibitor being developed for the treatment of rheumatoid arthritis (RA) and other inflammatory diseases. This work characterized upadacitinib population pharmacokinetics in healthy subjects and RA patients and the effects of covariates on upadacitinib exposure.

Methods Upadacitinib plasma concentrations $(n=6399)$ from 107 healthy subjects and 466 RA patients from three phase I and two 12-week RA phase IIb trials (1-48 mg immediate-release doses across studies) were analyzed using non-linear mixed-effects modeling. The models were qualified using bootstrap and stochastic simulations.

Results A two-compartment model with first-order absorption and elimination described upadacitinib pharmacokinetics. Estimates (95\% bootstrap confidence interval) for upadacitinib oral clearance, steady-state volume of distribution, absorption lag time, and mean absorption time were $39.7 \quad(37.8-41.5) \mathrm{L} / \mathrm{h}, \quad 210 \quad(196-231) \mathrm{L}, \quad 0.48$ $(0.47-0.49) \mathrm{h}$, and $0.08(0.04-0.12) \mathrm{h}$, respectively, for a typical healthy male. Matching on other covariates, a 16 and $32 \%$ higher upadacitinib area under the concentration-
\end{abstract}

Electronic supplementary material The online version of this article (doi:10.1007/s40262-017-0605-6) contains supplementary material, which is available to authorized users.

Ahmed A. Othman

ahmed.othman@abbvie.com

1 AbbVie Clinical Pharmacology and Pharmacometrics, AbbVie Deutschland GmbH \& Co. KG, Ludwigshafen am Rhein, Germany

2 AbbVie Clinical Pharmacology and Pharmacometrics, AbbVie, 1 North Waukegan Road, Bldg. AP31-3, North Chicago, IL 60064, USA time curve (AUC) was estimated for females relative to males, and for subjects with RA relative to healthy volunteers, respectively. Subjects with RA with mild or moderate renal impairment were estimated to have 16 and $32 \%$ higher upadacitinib AUC, respectively, compared with subjects with RA with normal renal function. Upadacitinib clearance was not correlated with body weight.

Conclusions Upadacitinib pharmacokinetics follow doseproportional, bi-exponential disposition. A slightly lower upadacitinib clearance is estimated in subjects with RA than in healthy volunteers, consistent with observations for other JAK inhibitors. Other covariates (weight, sex, mild or moderate renal impairment) are not associated with clinically relevant effects on upadacitinib exposure.

Trial Registration ClinicalTrials.gov (https://clinicaltrials. gov/) identifiers: NCT01741493, NCT02066389, and NCT01960855.

\section{Key Points}

A two-compartment model with first-order absorption and elimination best described upadacitinib pharmacokinetics.

The apparent clearance of upadacitinib is estimated to be $24 \%$ lower in RA patients than in healthy subjects.

Differences in body weight, sex, or renal function (mild or moderate renal impairment) do not result in clinically relevant effects on upadacitinib exposures. Further assessment of covariate effects are warranted using data from phase III studies when available. 


\section{Introduction}

Rheumatoid arthritis (RA) is a chronic systemic inflammatory disease that occurs in $0.5-1 \%$ of adults worldwide and represents a major cause of disability [1]. Despite the availability of various treatment options for RA, including biologic and non-biologic disease-modifying antirheumatic drugs (DMARDs), a significant proportion of patients either do not adequately respond or become refractory to available therapies [2]. Therefore, novel therapies are required to address the unmet need in the treatment of patients with RA.

Janus kinase (JAK) enzymes are tyrosine kinases that bind to the cytoplasmic region of cytokine receptors and are activated following the binding of cytokines to their cellular receptors [3]. The JAK enzyme family consists of four members, JAK1, JAK2, JAK3, and TYK1, which play important roles in signaling pathways of growth factors, hormones and various cytokines, including those involved in the pathogenesis of inflammatory diseases. It has been hypothesized that selectively inhibiting JAK1 can intercept the pathogenic cytokine signaling involved in RA while sparing other signaling pathways required for normal physiological functions [4].

Upadacitinib (ABT-494) is a selective JAK 1 inhibitor being developed for the treatment of patients with inflammatory diseases. Currently, upadacitinib is being evaluated in six global phase III studies in RA [5-10] and two phase III studies in psoriatic arthritis (PsA) [11, 12], in addition to phase II studies in Crohn's disease and atopic dermatitis $[13,14]$ and a combined phase II/III study in ulcerative colitis [15]. In phase I studies, upadacitinib was well-tolerated in single doses up to $48 \mathrm{mg}$ and multiple twice-daily doses up to $24 \mathrm{mg}$ administered as immediaterelease capsules [16]. Upadacitinib exposures were approximately dose proportional over the evaluated dose range and displayed no accumulation in plasma with multiple twice-daily dosing. Upadacitinib functional halflife was approximately $4 \mathrm{~h}$ and its mean terminal elimination half-life ranged from 6 to $16 \mathrm{~h}$. Upadacitinib is a non-sensitive substrate for cytochrome P450 (CYP) 3A, and in vitro studies suggest that CYP2D6 may be a minor contributor to its metabolism. Approximately $20 \%$ of upadacitinib dose is eliminated unchanged in urine [16]. Upadacitinib has moderate plasma protein binding $(<50 \%)$.

Upadacitinib was recently evaluated in two phase IIb studies in subjects with RA who were inadequate responders to methotrexate or anti-tumor necrosis factor (TNF) therapy $[17,18]$. The doses of upadacitinib evaluated in these studies ranged from 3 to $18 \mathrm{mg}$ twice daily, in addition to $24 \mathrm{mg}$ once daily in one study, administered as immediate-release capsules. Response, measured as the percentage of subjects meeting American College of Rheumatology 20\% improvement criteria (ACR20), at week 12 was higher with all evaluated upadacitinib doses compared with placebo, with the maximum efficacy reached at doses of 6 or $12 \mathrm{mg}$ twice daily. The efficacy of upadacitinib was also demonstrated on deeper response endpoints (ACR50 and ACR70, as well as Disease Activity Score 28 based on C-reactive protein [DAS-28CRP] low disease activity and remission). Upadacitinib showed an acceptable safety profile in these studies, which supported proceeding with large global phase III studies to confirm its efficacy and safety.

Population pharmacokinetic analyses of integrated data from multiple studies and patient cohorts provides a powerful tool to robustly characterize the pharmacokinetic parameters for a drug as well as the influence of subject characteristics on plasma exposures, which is necessary to inform the need for dose adjustment in certain subpopulations [19]. The aim of this work was to develop a population pharmacokinetic model for upadacitinib in healthy subjects and in adult subjects with RA, and to identify the effect of subject characteristics on upadacitinib plasma exposures.

\section{Methods}

\subsection{Participants and Design of Clinical Studies Analyzed}

The population pharmacokinetic analysis was performed using combined data from three phase I studies conducted in healthy subjects and subjects with RA and two phase II studies conducted in subjects with RA (Table 1). Upadacitinib was administered as immediate-release capsules in all studies included in the analysis. The study protocols were approved by the Institutional Review Boards/Ethics Committees of the study sites, and all the participants gave written informed consent prior to participation in the studies. The studies were conducted according to the International Conference on Harmonization Guidelines for Good Clinical Practice.

In the three phase I studies (studies 1, 2, and 3 in Table 1), healthy subjects were eligible to participate if they were 18-55 years of age, with a body mass index within $19-29 \mathrm{~kg} / \mathrm{m}^{2}$, and judged to be in good general health. In addition to healthy subjects, study 2 enrolled a cohort of subjects who were 18-75 years of age with a body mass index within $19-39 \mathrm{~kg} / \mathrm{m}^{2}$ at screening, had a diagnosis of RA for at least 6 months, and had been receiving a stable dose of methotrexate for at least 3 months. Study 3 enrolled subjects of Japanese or Chinese 
Table 1 Overview of phase I and II studies included in the upadacitinib population pharmacokinetic analysis

\begin{tabular}{|c|c|c|c|c|c|c|}
\hline Study & Population & $N^{\mathrm{a}}$ & $\begin{array}{l}\text { Study design } \\
\text { Pharmacokinetic sampling/subject }\end{array}$ & $\begin{array}{l}\text { Upadacitinib } \\
\text { immediate-release } \\
\operatorname{dose}(s)\end{array}$ & $\begin{array}{l}\text { Concomitant } \\
\text { methotrexate } \\
\text { (yes/no) }\end{array}$ & References \\
\hline \multicolumn{7}{|c|}{ Phase I } \\
\hline 1 & Healthy subjects & 56 & $\begin{array}{l}\text { Single dose, randomized, placebo-controlled } \\
17 \text { samples up to } 72 \mathrm{~h} \text { post-dose }\end{array}$ & $\begin{array}{l}1,3,6,12,24,36 \\
48 \mathrm{mg}\end{array}$ & No & [16] \\
\hline \multirow[t]{2}{*}{2} & Healthy subjects & 44 & $\begin{array}{l}\text { Multiple dose, randomized, placebo-controlled } \\
11 \text { samples up to } 12 \mathrm{~h} \text { post day } 1 \text { morning dose } \\
\text { and } 18 \text { samples up to } 72 \mathrm{~h} \text { post day } 14 \text { dose }\end{array}$ & $\begin{array}{l}3,6,12,24 \mathrm{mg} \\
\text { bid for } 14 \text { days }\end{array}$ & No & [16] \\
\hline & $\begin{array}{l}\text { Subjects with mild to } \\
\text { moderate RA }\end{array}$ & 14 & $\begin{array}{l}\text { Multiple dose, randomized, placebo-controlled } \\
11 \text { samples up to } 12 \mathrm{~h} \text { post days } 1 \text { and } 26 \\
\text { morning doses and } 17 \text { samples up to } 48 \mathrm{~h} \text { post } \\
\text { day } 27 \text { dose }\end{array}$ & $6,12,24 \mathrm{mg}$ & Yes & \\
\hline \multirow[t]{2}{*}{3} & $\begin{array}{l}\text { Healthy Japanese and } \\
\text { Chinese subjects }\end{array}$ & 45 & $\begin{array}{l}\text { Single dose, randomized, placebo-controlled } \\
17 \text { samples up to } 72 \text { h post dose }\end{array}$ & $3,6,24 \mathrm{mg}$ & No & - \\
\hline & & & $\begin{array}{l}\text { Multiple dose, randomized, placebo-controlled } \\
11 \text { samples up to } 12 \mathrm{~h} \text { post day } 1 \text { morning dose } \\
\text { and } 18 \text { samples up to } 72 \mathrm{~h} \text { post day } 14 \text { dose }\end{array}$ & $\begin{array}{l}18 \mathrm{mg} \text { bid for } \\
14 \text { days }\end{array}$ & & \\
\hline \multicolumn{7}{|c|}{ Phase II } \\
\hline 4 & $\begin{array}{l}\text { Subjects with moderate to } \\
\text { severe active RA (MTX- } \\
\text { IR) }\end{array}$ & 300 & $\begin{array}{l}\text { Randomized, placebo-controlled, dose-ranging } \\
\text { Single predose trough sample at weeks } 2,4,6,8 \text {, } \\
\text { and } 12 \\
\text { Samples at } 1,2,3 \mathrm{~h} \text { after the morning dose on } \\
\text { day } 1 \text { and week } 8 \text { in } \sim 30 \% \text { of subjects }\end{array}$ & $\begin{array}{l}3,6,12 \text {, and } \\
18 \mathrm{mg} \text { bid and } \\
24 \mathrm{mg} \mathrm{qd}\end{array}$ & Yes & [18] \\
\hline 5 & $\begin{array}{l}\text { Subjects with moderate to } \\
\text { severe active RA (anti- } \\
\text { TNF IR) }\end{array}$ & 276 & $\begin{array}{l}\text { Randomized, placebo-controlled, dose-ranging } \\
\text { Single sample at weeks } 2,4,6,8 \text {, and } 12 \\
\text { Samples at } 1,2,3 \mathrm{~h} \text { after the morning dose on } \\
\text { day } 1 \text { and week } 8 \text { in } \sim 30 \% \text { of subjects }\end{array}$ & $\begin{array}{c}3,6,12, \text { and } \\
18 \mathrm{mg} \text { bid }\end{array}$ & Yes & [17] \\
\hline
\end{tabular}

bid twice daily, $R A$ rheumatoid arthritis, MTX-IR methotrexate inadequate responders, anti-TNF IR anti-tumor necrosis factor inadequate responders, $q d$ once daily

${ }^{\text {a }}$ Total number of subjects enrolled in the study

origin. In all three studies, individuals were excluded if they had any clinically significant condition (except stable RA in the patient cohort of study 2), abnormalities, infection, or any clinically significant findings at screening, as determined by the principal investigator. Inhibitors or inducers of drug-metabolizing enzymes were not allowed within 30 days of enrollment. Subjects were randomized to receive single doses of upadacitinib or placebo in study 1 , multiple doses of upadacitinib or placebo for 14 days in healthy subjects and 26 days in subjects with RA in study 2 , and single or multiple doses of upadacitinib or placebo for 14 days in study 3 .

In the two phase II studies (studies 4 and 5), adult males and females 18 years of age or older who had active RA for at least 3 months were enrolled in the studies. Subjects were enrolled in study 4 if they had inadequate response to methotrexate, and were excluded if they had received any biologic RA therapy. Subjects were enrolled in study 5 if they had inadequate response to at least one prior anti-TNF therapy. In both studies 4 and 5, subjects were excluded if they had an estimated glomerular filtration rate (eGFR) of $<40 \mathrm{~mL} / \mathrm{min} / 1.73 \mathrm{~m}^{2}$ or serum aspartate transaminase (AST) or alanine transaminase (ALT) $>1.5 \times$ upper limit of normal at screening. Strong inhibitors or inducers of CYP3A were prohibited throughout both studies. Subjects in each study were randomized to receive different doses of upadacitinib or placebo for 12 weeks. Further details on the design and inclusion and exclusion criteria of studies 4 and 5 have been previously reported $[17,18]$.

In studies 1-3, serial blood samples were collected from each subject over the time periods delineated in Table 1. In studies 4 and 5, a single predose blood sample was collected from each subject at each study visit. In approximately $30 \%$ of subjects in studies 4 and 5, blood samples were collected at 1,2 , and $3 \mathrm{~h}$ after dose at the day 1 and week 8 visits. Plasma concentrations of upadacitinib were 
determined using a validated liquid chromatography method with mass spectrometric detection at AbbVie (North Chicago, IL, USA), as previously described [16].

Only $4 \%$ of upadacitinib concentrations for samples collected after initiation of dosing were found to be below the lower limit of quantitation (LLOQ) in the dataset. For each subject, concentrations below the LLOQ during the dosing period, and the first concentration below the LLOQ after the last dose, were imputed with 1/2 LLOQ [20]; subsequent concentrations below the LLOQ after the last dose were censored in the analysis. Upadacitinib concentrations from the phase II studies in RA were plotted versus time since the last dose for each patient individually to identify potential outliers. Only $0.9 \%$ of all dataset concentrations were flagged as clear outliers that likely resulted from inaccurate dosing records and were excluded from the analysis.

\subsection{Pharmacokinetic Model Development}

A non-linear mixed-effects modeling approach was utilized to analyze upadacitinib concentration versus time data. NONMEM $^{\circledR} 7.3$ software (ICON Development Solutions, Hanover, MD, USA) was utilized.

The population pharmacokinetic model was built in a stepwise manner. The structural model was developed first, and then models for intersubject variability (ISV) were added. The ISV was modeled using exponential error models:

$P_{n i}=\theta_{n} \cdot \exp \left(\eta_{n i}\right)$,

where $P_{n i}, \theta_{n}$, and $\eta_{n i}$ are the individual parameter estimate, population parameter estimate, and intersubject random effect, respectively, for subject $i$ and parameter $n$. The $\eta_{n}$ values were assumed to be normally distributed with a mean of 0 and a variance of $\omega_{n}{ }^{2}$.

The residual error was assumed to be a combination of additive and proportional error terms:

$C_{i j}=\widehat{C}_{i j} \cdot\left(1+\varepsilon_{1 i j}\right)+\varepsilon_{2 i j}$,

where $C_{i j}$ is the measured plasma concentration for subject $i$ at time $j, \hat{C}_{i j}$ is the corresponding model-predicted plasma concentration, and $\varepsilon_{1 i j}$ and $\varepsilon_{2 i j}$ are normally distributed independent residual random error terms with a mean of zero and variances of $\sigma^{2}$ :

$\varepsilon_{n} \sim N\left(0, \sigma_{n}^{2}\right)$.

The following covariates were evaluated: (1) for upadacitinib apparent clearance (CL/F): population (subjects with RA versus healthy subjects), baseline serum bilirubin concentration, baseline serum creatinine, baseline creatinine clearance, baseline eGFR, body weight, age, baseline serum AST concentration, baseline serum ALT concentration, baseline DAS28-CRP, baseline highsensitivity C-reactive protein (hsCRP) serum concentration, upadacitinib dose, sex, race, baseline alcohol use, baseline tobacco use, concomitant use of pH-modifying medications (e.g. proton pump inhibitors or antacids), concomitant use of CYP3A inhibitors, concomitant use of CYP2D6 inhibitors, and CYP2D6 metabolic phenotype (determined based on CYP2D6 genotype); (2) for the apparent volume of distribution of the central compartment $\left(V_{\mathrm{c}} / \mathrm{F}\right)$ : population, sex, race, body weight, upadacitinib dose, and age; (3) for the first-order absorption rate $\left(K_{\mathrm{a}}\right)$ : population, sex, race, baseline alcohol use, upadacitinib dose, age, and concomitant use of $\mathrm{pH}$ modifying medications; and (4) for relative oral bioavailability: upadacitinib dose. For healthy subjects, missing baseline values of DAS28-CRP and hsCRP were imputed as 0.96 and $0.15 \mathrm{mg} / \mathrm{L}$, respectively. For all other missing continuous covariate information, the population median value was assigned. Continuous covariates were evaluated using power models (centered on the median values in the datasets; referred to thereafter as the reference values), whereas categorical covariates were evaluated using indicator variables. The covariate that resulted in the largest drop in the objective function value (OFV) in a univariate covariate search was included in the model first, and then the process was repeated to identify the second covariate to be included in the model, and so forth.

The pharmacokinetic models were fit to the data using the first-order conditional estimation with interaction (FOCEI). The stochastic approximation expectation maximization (SAEM) algorithm with importance sampling employed within NONMEM was utilized at the last step to facilitate incorporation of interoccasion variability (IOV) on the lag time, as described in detail in the "Results" section. The likelihood ratio test was used for hypothesis testing to discriminate among alternative nested models. Since the OFV provided by NONMEM is approximately Chi square distributed, the differences between OFV values were used to guide model building. When comparing nested models, one additional model parameter, corresponding to one degree of freedom in the higher-order model, was considered significant if it lowered the OFV by more than 6.63 , corresponding to $p<0.01$.

The significance of covariates was evaluated through forward inclusion and backward elimination procedures using the likelihood ratio test with $p$ value thresholds of $p<0.01$ and $p<0.001$, respectively. This was implemented on the last model estimated with FOCEI prior to inclusion of IOV with SAEM and importance sampling since the implementation of importance sampling is associated with Monte Carlo noise in the objective function that can, in certain cases, confound statistical comparisons. In addition 
to the likelihood ratio test, selection of the models was based on the following criteria: the observed and predicted plasma concentrations from the preferred model were more randomly distributed across the line of unity (a straight line with zero intercept and a slope of one) than alternative models, the conditional weighted residuals of the preferred model showed less systematic bias than alternative models, the preferred model showed physiologically reasonable and statistically significant estimates (95\% confidence intervals [CIs] did not include zero) of the parameters and their standard errors, and visual predictive checks (VPCs) showed appropriate characterization of observed trends and variability.

VPCs and prediction-corrected VPCs [21] were examined based on 400 simulated replicates of the dataset generated using NONMEM and Perl Speaks NONMEM (PSN 4, http://psn.sourceforge.net/index.php, Uppsala University, Uppsala, Sweden). In order to estimate CIs of the model parameters, 200 bootstrap replicates were constructed by randomly sampling $N$ subjects from the original dataset with replacement, where $N$ was the number of subjects in the original dataset. Model parameters were estimated for each bootstrap replicate and the resulting values were used to estimate medians and CIs. Bootstrap statistics were based on successfully completed runs.

Simulations with 200 replicates using the demographics of the RA patient population from the two phase II studies were performed to explore the impact of covariates on upadacitinib area under the concentration-time curve (AUC) and maximum concentration $\left(C_{\max }\right)$ at steady state. Parameter uncertainty was incorporated using the covariance matrix obtained from the bootstrap analysis. While exploring the impact of one of the identified covariates on exposure, other significant covariates were fixed to the reference values.

\section{Results}

\subsection{Data Summary and Demographics}

Baseline characteristics for subjects included in the analyses are summarized in Table 2. A total of 6399 plasma concentrations from 107 healthy volunteers and 466 RA patients were included in the analysis.

\subsection{Upadacitinib Pharmacokinetic Model}

The key steps in the model building history and the associated changes in the NONMEM objective function are presented in electronic supplementary Table S1. A twocompartment model with absorption lag time (ALAG1) was found to be the best structural model among different models tested. The use of transit compartments for describing the absorption process did not provide a significantly better fit compared with the model with ALAG1. Inclusion of log-normally distributed ISV on $\mathrm{CL} / \mathrm{F}, V_{\mathrm{c}} / \mathrm{F}$, and $K_{\mathrm{a}}$ provided the best fit and was hence chosen for further development. No meaningful correlation $(<12 \%)$ was found between the ISV parameters.

Covariates were evaluated using the forward inclusion/ backward elimination procedure, as described in the "Methods" section. Covariates included in the forward inclusion steps were subject population, creatinine clearance and sex on $\mathrm{CL} / \mathrm{F}$, and sex and total body weight on $V_{\mathrm{c}} / \mathrm{F}$. All included covariates remained statistically significant in the backward elimination steps, and therefore none were eliminated. A significant correlation was not found between any of the evaluated concomitant medications of interest (CYP3A inhibitors, CYP2D6 inhibitors, or $\mathrm{pH}-$ modifying medications) and upadacitinib pharmacokinetic parameters. Additionally, CYP2D6 phenotype (as determined from the pharmacogenetic analysis) was not correlated to upadacitinib clearance.

A model with different ISV variances for each of CL/F and $V_{\mathrm{c}} / \mathrm{F}$ for subjects with RA versus healthy subjects provided a significant improvement in the model fit and was chosen for further development. Stepwise backward elimination of covariates was repeated after inclusion of different ISV variances on $\mathrm{CL} / \mathrm{F}$ and $V_{\mathrm{c}} / \mathrm{F}$ for RA patients versus healthy subjects (electronic supplementary Table S2). Statistical significance of all covariates included in the model was reconfirmed in this step.

The addition of log-normal IOV on ALAG1 for the visits of the phase II studies in subjects with RA was evaluated to account for potential inaccuracies in recording time of dosing or sampling. The inclusion of log-normal IOV on ALAG1 resulted in a strong drop of the OFV ( $\sim 1800$ points). However, the empirical Bayes estimates of the IOV on ALAG1 exhibited a heavy right-tailed distribution, suggesting that the IOV is not log-normally distributed. To address this finding, a right-skewed box-cox transformation of the form

$\eta_{i \_ \text {transformed }}=\frac{\left(\left(\mathrm{e}^{\eta_{i}}\right)^{\theta}-1\right)}{\theta}$

with skewness parameter $\theta>0$, was applied to the IOV [22]. Models with different skewness parameters ranging from 0.25 to 16 were tested to improve the predictioncorrected VPCs compared with the previous model without IOV. All transformations tested led to a significant drop in the OFV. An increase in the skewness parameter resulted in increasing agreement of observed and simulated data in prediction-corrected VPCs, and the final pharmacokinetics model was selected with a skewness parameter value of 8 , 
Table 2 Demographics summary for the population pharmacokinetic analysis dataset

\begin{tabular}{|c|c|c|c|c|c|c|c|}
\hline Characteristic & $\begin{array}{l}\text { Study } 1 \\
\text { HVs }\end{array}$ & $\begin{array}{l}\text { Study } 2 \\
\text { HVs }\end{array}$ & $\begin{array}{l}\text { Study } 2 \\
\text { RA patients }\end{array}$ & $\begin{array}{l}\text { Study } 3 \\
\text { HVs }\end{array}$ & $\begin{array}{l}\text { Study } 4 \\
\text { RA patients }\end{array}$ & $\begin{array}{l}\text { Study } 5 \\
\text { RA patients }\end{array}$ & Total \\
\hline$N$ & 42 & 32 & 10 & 33 & 242 & 214 & 573 \\
\hline \multicolumn{8}{|l|}{$\operatorname{Sex}[n(\%)]$} \\
\hline Male & $35(83)$ & $29(91)$ & $6(60)$ & $33(100)$ & $48(20)$ & $47(22)$ & $198(35)$ \\
\hline Female & 7 (17) & $3(9)$ & $4(40)$ & 0 & $194(80)$ & 167 (78) & $375(65)$ \\
\hline \multicolumn{8}{|l|}{ Race $[n(\%)]$} \\
\hline White & $30(71)$ & $12(38)$ & $9(90)$ & 0 & $236(98)$ & $190(89)$ & $477(83)$ \\
\hline Black & $10(24)$ & $9(28)$ & $1(10)$ & 0 & $3(1)$ & $18(8)$ & $41(7)$ \\
\hline Asian & $1(2)$ & 0 & 0 & $33(100)$ & 0 & $3(1)$ & $37(6)$ \\
\hline Other & $1(2)$ & $11(34)$ & 0 & 0 & $3(1)$ & $3(1)$ & $18(3)$ \\
\hline $\begin{array}{l}\text { Age, years }[\text { mean } \pm \mathrm{SD} \\
\text { (range)] }\end{array}$ & $\begin{array}{l}31 \pm 10 \\
(19-54)\end{array}$ & $\begin{array}{r}33 \pm 9.9 \\
(22-56)\end{array}$ & $\begin{array}{r}59 \pm 8.7 \\
(44-70)\end{array}$ & $\begin{array}{l}38 \pm 11 \\
\quad(22-55)\end{array}$ & $\begin{array}{l}55 \pm 12 \\
\quad(19-82)\end{array}$ & $\begin{array}{l}57 \pm 12 \\
\quad(26-85)\end{array}$ & $\begin{array}{l}52 \pm 15 \\
\quad(19-85)\end{array}$ \\
\hline $\begin{array}{l}\text { Weight, kg [mean } \pm \mathrm{SD} \\
\text { (range)] }\end{array}$ & $\begin{array}{l}74 \pm 10 \\
(52-92)\end{array}$ & $\begin{array}{l}76 \pm 10 \\
(57-93)\end{array}$ & $\begin{array}{l}79 \pm 16 \\
\quad(58-101)\end{array}$ & $\begin{array}{r}69 \pm 9.3 \\
(53-90)\end{array}$ & $\begin{array}{l}75 \pm 15 \\
\quad(44-122)\end{array}$ & $\begin{array}{l}79 \pm 17 \\
(42-134)\end{array}$ & $\begin{array}{l}76 \pm 15 \\
\quad(42-134)\end{array}$ \\
\hline RA $[n(\%)]$ & 0 & 0 & $10(100)$ & 0 & $242(100)$ & $214(100)$ & $466(81)$ \\
\hline $\begin{array}{l}\text { hsCRP, mg/L [mean } \pm \mathrm{SD} \\
\text { (range)] }\end{array}$ & $\begin{array}{l}1.64 \pm 1.8 \\
(0.06-6.8)\end{array}$ & - & $\begin{array}{l}5.9 \pm 8.3 \\
\quad(0.14-28)\end{array}$ & - & $\begin{array}{l}13 \pm 16 \\
(0.07-90)\end{array}$ & $\begin{array}{l}14 \pm 20 \\
\quad(0.21-135)\end{array}$ & $\begin{array}{l}12 \pm 18 \\
(0.06-135)\end{array}$ \\
\hline $\begin{array}{l}\text { DAS28-CRP [mean } \pm \text { SD } \\
\quad \text { (range)] }\end{array}$ & - & - & - & - & $\begin{array}{l}5.6 \pm 0.97 \\
(3-7.9)\end{array}$ & $\begin{array}{l}5.8 \pm 0.92 \\
\quad(3.6-8)\end{array}$ & $\begin{array}{l}5.7 \pm 0.95 \\
(3-8)\end{array}$ \\
\hline $\begin{array}{l}\text { eGFR, } \mathrm{mL} / \mathrm{min} / 1.73 \mathrm{~m}^{2} \\
{[\text { mean } \pm \mathrm{SD} \text { (range)] }}\end{array}$ & $\begin{array}{l}95 \pm 13 \\
\quad(66-125)\end{array}$ & $\begin{array}{l}92 \pm 14 \\
\quad(65-120)\end{array}$ & $\begin{array}{l}83 \pm 17 \\
\quad(52-101)\end{array}$ & $\begin{array}{l}93 \pm 14 \\
\quad(63-121)\end{array}$ & $\begin{array}{l}92 \pm 23 \\
\quad(41-168)\end{array}$ & $\begin{array}{l}93 \pm 26 \\
(37-184)\end{array}$ & $\begin{array}{l}92 \pm 23 \\
\quad(36-184)\end{array}$ \\
\hline $\begin{array}{l}\mathrm{CrCL}, \mathrm{mL} / \mathrm{min}[\text { mean } \pm \mathrm{SD} \\
\quad \text { (range)] }\end{array}$ & $\begin{array}{l}118 \pm 19 \\
(86-174)\end{array}$ & $\begin{array}{l}113 \pm 24 \\
(76-176)\end{array}$ & $\begin{array}{l}95 \pm 21 \\
\quad(65-128)\end{array}$ & $\begin{array}{l}105 \pm 19 \\
\quad(65-148)\end{array}$ & $\begin{array}{c}108 \pm 34 \\
(41-211)\end{array}$ & $\begin{array}{c}111 \pm 38 \\
\quad(41-241)\end{array}$ & $\begin{array}{c}110 \pm 34 \\
\quad(41-241)\end{array}$ \\
\hline \multicolumn{8}{|l|}{ CYP2D6 phenotype $[n(\%)]$} \\
\hline EM & $38(90)$ & 19 (59) & 0 & 0 & $161(67)$ & $122(57)$ & 340 (59) \\
\hline IM & $3(7)$ & $5(16)$ & 0 & $1(3)$ & $12(5)$ & $13(6)$ & $34(6)$ \\
\hline UM & 0 & 0 & 0 & 0 & $5(2)$ & $2(1)$ & $7(1)$ \\
\hline PM & $1(2)$ & $1(3)$ & 0 & 0 & $14(6)$ & $13(6)$ & $29(5)$ \\
\hline NA & 0 & $7(22)$ & $10(100)$ & 32 (97) & $50(21)$ & $64(30)$ & $163(28)$ \\
\hline
\end{tabular}

$C r C L$ creatinine clearance, $e G F R$ estimated glomerular filtration rate, $R A$ rheumatoid arthritis, $h s C R P$ high-sensitivity C-reactive protein, $D A S 28-C R P$ Disease Activity Score 28 based on C-reactive protein, $E M$ extensive metabolizer, $I M$ intermediate metabolizer, $U M$ ultra-rapid metabolizer, $P M$ poor metabolizer, $N A$ classification not available, $C Y P$ cytochrome $\mathrm{P} 450, S D$ standard deviation

beyond which no clear improvement of the VPCs could be observed. The SAEM algorithm with importance sampling in NONMEM was used to estimate the parameters for the final model incorporating the IOV on ALAG1 (model with IOV did not successfully converge using FOCE).

A bootstrap analysis confirmed the robustness of the parameter estimates, and the $95 \%$ CIs of the covariate parameter estimates confirmed statistical significance of the included covariates (Table 3). Individual model-predicted concentrations showed good agreement with observations (Fig. 1). The conditional weighted residuals plotted versus time since last dose or population predicted upadacitinib plasma concentrations showed no systematic trend, indicating that the final model was appropriately specified (Fig. 2). The ISVs for CL/F and $V_{\mathrm{c}} / \mathrm{F}$ did not show residual correlation with significant covariates (results not shown), indicating that the covariate effects were appropriately accounted for in the final model. VPCs stratified by dose group and patient population (Fig. 3 and electronic supplementary Figs. S1, S2, and S3), as well as a prediction-corrected VPC (not shown), showed good agreement between simulated and observed data.

Upadacitinib pharmacokinetic parameter estimates from the final model are shown in Table 3. In healthy volunteers using reference covariate values (male, body weight of $74 \mathrm{~kg}$, and creatinine clearance of $107 \mathrm{~mL} / \mathrm{min}$ ), the population estimates $(95 \% \mathrm{CI})$ for upadacitinib CL/F, apparent steady-state volume of distribution (calculated as the sum of $V_{\mathrm{c}} / \mathrm{F}$ and $\left.V_{\mathrm{p}} / \mathrm{F}\right)$, ALAG1, and mean absorption time (calculated as $1 / K_{\mathrm{a}}$ ) were $39.7(37.8-41.5) \mathrm{L} / \mathrm{h}, 210$ (196-231) L, $0.48(0.47-0.49) \mathrm{h}$, and $0.08(0.04-0.12) \mathrm{h}$, respectively. 
Table 3 Upadacitinib population pharmacokinetic parameter estimates from the final model and results of the bootstrap analysis

\begin{tabular}{|c|c|c|c|c|}
\hline \multirow[t]{2}{*}{ Parameter } & \multirow{2}{*}{$\begin{array}{l}\text { Population analysis } \\
\text { Point estimate } \\
\text { (\%RSE) }\end{array}$} & \multicolumn{3}{|c|}{ Bootstrap analysis ${ }^{\mathrm{a}}$} \\
\hline & & Mean & Median & $95 \% \mathrm{CI}$ \\
\hline $\mathrm{CL} / \mathrm{F}(\mathrm{L} / \mathrm{h})$ & $39.7(2)$ & 39.8 & 39.8 & $37.8-41.5$ \\
\hline$V_{\mathrm{c}} / \mathrm{F}(\mathrm{L})$ & $146(2.2)$ & 145 & 145 & $139-152$ \\
\hline$K_{\mathrm{a}}(1 / \mathrm{h})^{\mathrm{b}}$ & 12.3 & 20.1 & 12.2 & $8.37-26.0$ \\
\hline ALAG1 (h) & $0.48(0.017)$ & 0.48 & 0.48 & $0.47-0.49$ \\
\hline$V_{\mathrm{p}} / \mathrm{F}(\mathrm{L})$ & $64.3(1.1)$ & 65.4 & 64.6 & $53.9-81.6$ \\
\hline $\mathrm{Q} / \mathrm{F}(\mathrm{L} / \mathrm{h})$ & $3.23(2.5)$ & 3.24 & 3.23 & $2.79-3.77$ \\
\hline $\mathrm{ISV}_{\mathrm{CL}}$ in healthy subjects (\%) & $16(8.5)$ & 16 & 16 & 14-19 \\
\hline $\mathrm{ISV}_{\mathrm{CL}}$ in subjects with RA (\%) & $26(2.9)$ & 26 & 26 & $23-29$ \\
\hline $\mathrm{ISV}_{V_{c}}$ in healthy subjects (\%) & $14(14)$ & 14 & 14 & $10-17$ \\
\hline $\mathrm{ISV}_{V_{c}}$ in subjects with RA (\%) & $27(5.5)$ & 26 & 26 & $21-31$ \\
\hline $\operatorname{ISV}_{K_{\mathrm{a}}}(\%)$ & $150(39)$ & 162 & 152 & $128-227$ \\
\hline Additive residual error SD (ng/mL) & $0.18(13)$ & 0.17 & 0.17 & $0.03-0.26$ \\
\hline Proportional residual error SD $(\%)$ & $31(13)$ & 31 & 31 & $29-34$ \\
\hline Ratio of $\mathrm{CL} / \mathrm{F}$ in RA patients compared with healthy subjects $(\theta 1)^{\mathrm{c}}$ & $0.76(2.9)$ & 0.76 & 0.76 & $0.7-0.82$ \\
\hline Ratio of $\mathrm{CL} / \mathrm{F}$ in females compared with males $(\theta 2)^{\mathrm{c}}$ & $0.86(2.8)$ & 0.86 & 0.86 & $0.79-0.93$ \\
\hline Exponent for the effect of CrCL on CL/F $(\theta 3)^{c}$ & $0.32(11)$ & 0.32 & 0.32 & $0.23-0.42$ \\
\hline Ratio of $V_{\mathrm{c}} / \mathrm{F}$ in females compared with males $(\theta 4)^{\mathrm{d}}$ & $0.75(1.6)$ & 0.75 & 0.75 & $0.7-0.8$ \\
\hline Exponent for the effect of body weight on $V_{c} / F(\theta 5)^{d}$ & $0.50(15.8)$ & 0.50 & 0.50 & $0.36-0.67$ \\
\hline$\omega_{\text {IOV }}^{2}$ on ALAG1 (phase II study visits only) & $0.08(1.5)$ & 0.079 & 0.079 & $0.07-0.09$ \\
\hline $\begin{array}{l}\text { Box-cox skewness parameter for the distribution of IOV on ALAG1 (phase II studies } \\
\text { only) }\end{array}$ & 8 (fixed) & - & - & - \\
\hline
\end{tabular}

ALAG1 absorption lag time, $B W$ body weight, $C I$ confidence interval, $C L / F$ apparent clearance, $C r C L$ creatinine clearance, $I O V$ interoccasion variability, $I S V$ intersubject variability, $K_{a}$ absorption rate constant, $Q / F$ apparent intercompartmental clearance, $R A$ rheumatoid arthritis, $R S E$ relative standard error, $S A E M$ stochastic approximation expectation maximization, $S D$ standard deviation, $S Q R T$ square root, $V_{c} / F$ apparent volume of distribution of the central compartment, $V_{p} / F$ apparent volume of distribution of the peripheral compartment

$\%$ ISV was calculated as $\operatorname{SQRT}\left(\omega^{2}\right) \times 100$

RSEs obtained from S-matrix provided by SAEM

a 188 successful runs out of 200

${ }^{\mathrm{b}} K_{\mathrm{a}}$ was modeled as an exponent of an estimated parameter theta (point estimate of 2.51 with RSE of $5.3 \%$ )

${ }^{\mathrm{c}}$ Typical clearance for a subject with a particular covariate combination $=\mathrm{CL} / \mathrm{F} \times \theta 1 \times \theta 2 \times(\mathrm{CrCL} / 107 \mathrm{~mL} / \mathrm{min})^{\theta 3}$, where $\theta 1=1$ for healthy volunteers and $\theta 2=1$ for males

${ }^{\mathrm{d}}$ Typical volume of central compartment for a subject with a particular covariate combination $=V_{\mathrm{c}} \times \theta 4 \times(\mathrm{BW} / 74 \mathrm{~kg})^{\theta 5}$, where $\theta 4=1 \mathrm{for}$ males

\subsection{Effect of Statistically Significant Covariates on Upadacitinib Exposures}

Simulations were performed to explore the impact of covariates on upadacitinib AUC and $C_{\max }$ at steady state. Subjects with RA were estimated to have a $32 \%$ higher upadacitinib AUC than healthy volunteers. Upadacitinib clearance correlation with sex was statistically significant but not clinically relevant, with a $16 \%$ estimated higher upadacitinib AUC in females compared with males. Renal function was estimated to have a modest effect on upadacitinib exposure, with a 16 and $32 \%$ higher upadacitinib AUC in subjects with RA with mild or moderate renal impairment, respectively, compared with RA subjects with normal renal function (Fig. 4).

\section{Discussion}

Upadacitinib is currently being evaluated in phase III trials for the treatment of RA and PsA, and in phase II studies in Crohn's disease, atopic dermatitis, and ulcerative colitis. We present in this report a population model that integrates available pharmacokinetic data across upadacitinib phase I studies and phase II RA studies. Data included in this analysis encompass a wide range of upadacitinib 

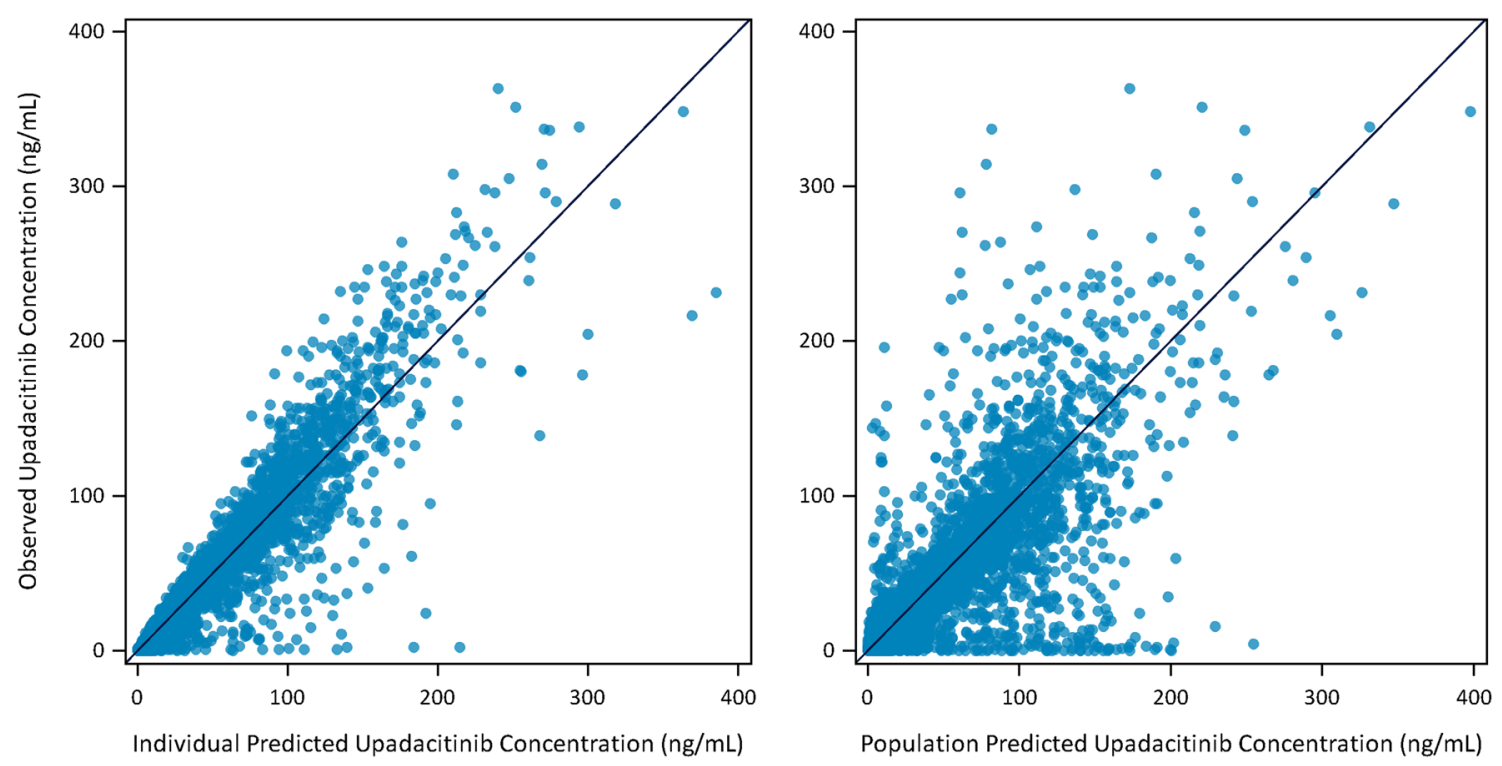

Fig. 1 Individual and population-predicted upadacitinib concentration versus observed upadacitinib concentration
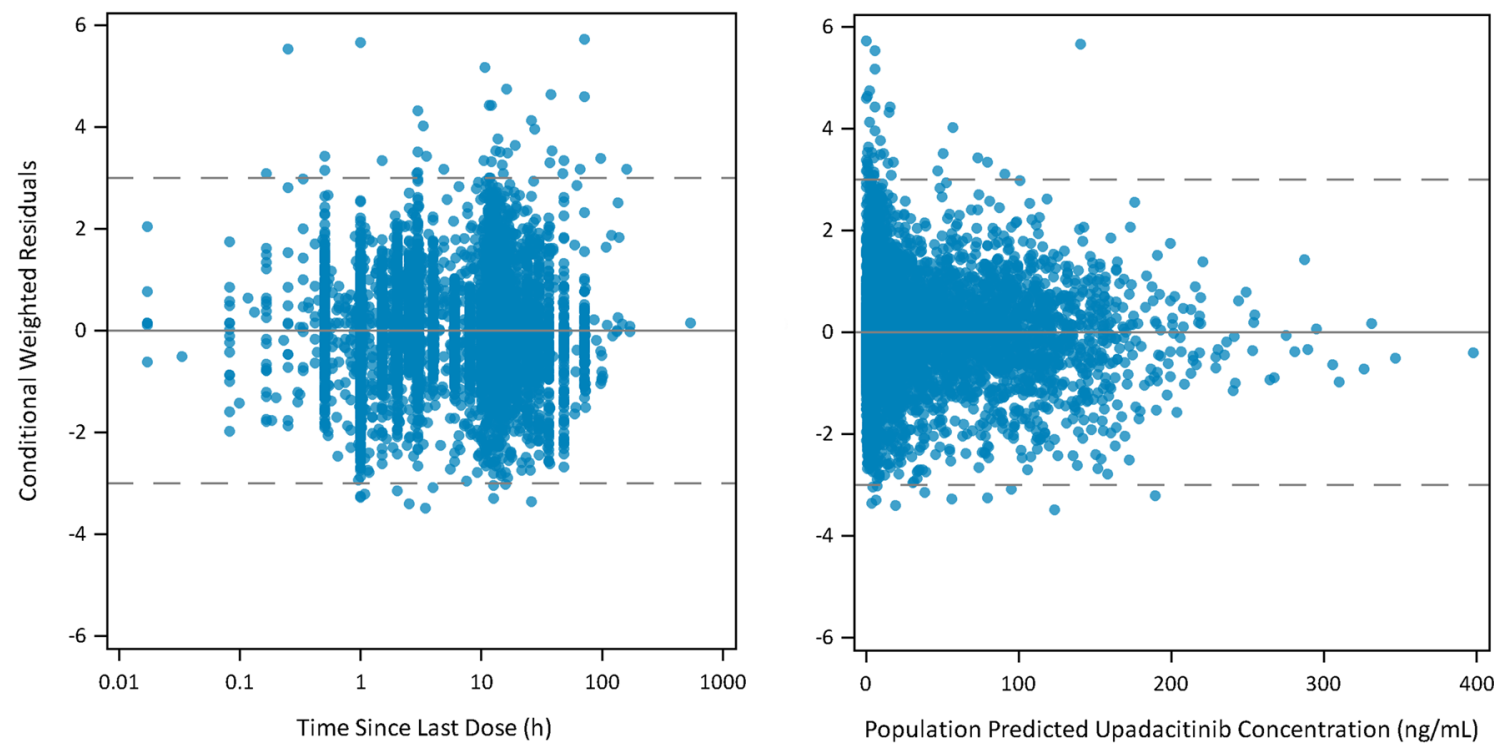

Fig. 2 Conditional weighted residuals versus time since last dose and versus population-predicted upadacitinib concentration

immediate-release doses (single doses of $1-48 \mathrm{mg}$ and multiple twice-daily doses of 3-24 mg, in addition to $24 \mathrm{mg}$ once-daily doses), different subject populations (healthy subjects and subjects with RA), and a relatively wide range of baseline characteristics (Table 2).

A two-compartment model with first-order absorption and elimination adequately characterized upadacitinib pharmacokinetics. Upadacitinib pharmacokinetics did not deviate significantly from linearity with respect to dose (dose was not found to significantly impact upadacitinib pharmacokinetic parameters), which is in agreement with previously reported non-compartmental analyses using single and multiple-dose data in healthy subjects [16].
Upadacitinib typical CL/F in subjects with RA is estimated to be $24 \%$ lower than in healthy subjects, leading to a 32\% higher AUC estimate. This population estimate of the difference in CL/F combining the data from phase I and II trials is in agreement with a previous non-compartmental analysis utilizing a small subset of the analyzed data in the present report (phase I, study 2) [16]. Oral clearance values for other JAK inhibitors (tofacitinib and filgotinib) have been reported to be lower in subjects with RA compared with healthy subjects $[24,25]$, similar to our observation for upadacitinib. The small difference in upadacitinib CL/F between subjects with RA and healthy subjects could not be explained by other covariates evaluated in the present 


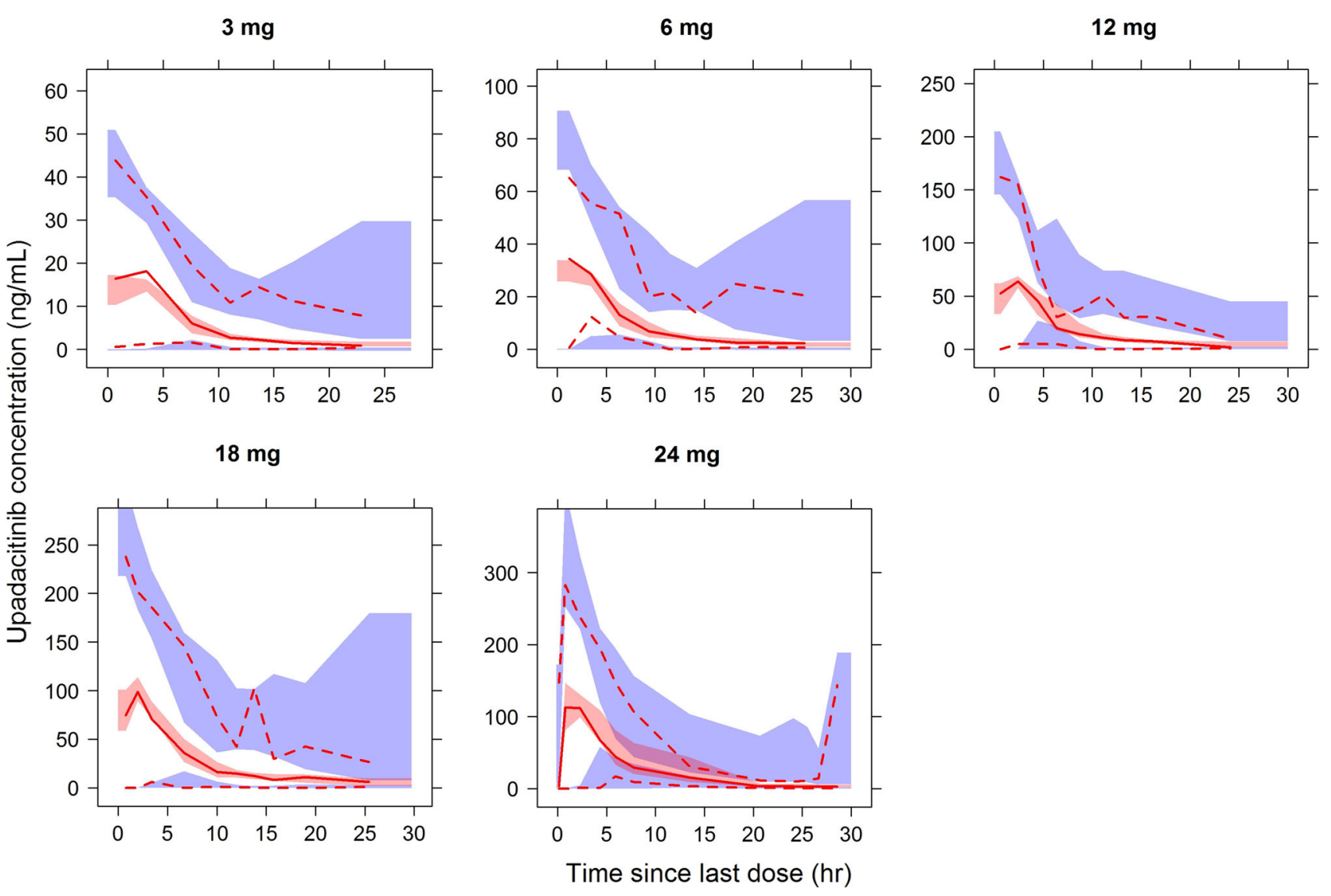

Fig. 3 Visual predicted check for observed and model-simulated upadacitinib plasma concentrations versus time since last dose for subjects with rheumatoid arthritis, stratified by dose. The solid red line represents the median observed plasma concentration, and the shaded red area represents a simulation-based 95\% confidence

analysis. Subjects with RA have elevated levels of interleukin-6, a proinflammatory cytokine that has been reported to suppress CYP3A metabolic activity, one of the routes contributing to upadacitinib clearance [26, 27]. In the analysis, inclusion of population (RA versus healthy volunteers) as a covariate on upadacitinib clearance was more statistically significant than inclusion of serum hsCRP, a marker that is significantly elevated in systemic inflammation. Once population was included in the model, no significant correlation between hsCRP and upadacitinib clearance could be detected. It is plausible that the difference observed in CL/F is due to a combination of factors (e.g. older age, lower metabolic capacity, and possibly inflammation) presented collectively in the RA population, leading to statistically significantly different CL/F compared with healthy subjects.

Creatinine clearance, but not serum creatinine, was statistically significantly correlated with upadacitinib CL/F (electronic supplementary Table S1). The range of creatinine clearance for subjects included in the analysis dataset was $42-241 \mathrm{~mL} / \mathrm{min}$. RA patients with mild and moderate interval for the median. The observed 5 and $95 \%$ percentiles are presented with dashed red lines, and the $95 \%$ confidence intervals for the corresponding model-predicted percentiles are shown as shaded blue areas

renal impairment, within the evaluated range, are estimated to have, on average, 16 and 32\% higher upadacitinib AUC compared with subjects with normal renal function (Fig. 4). This effect is considered modest and does not warrant dose adjustment in subjects within this range of creatinine clearance, and is in agreement with the small percentage of upadacitinib dose $(\sim 20 \%)$ eliminated unchanged in urine [16]. A dedicated phase I renal impairment study is currently ongoing and results from this study will further inform the findings from this population pharmacokinetic analysis.

Upadacitinib CL/F and $\mathrm{V}_{\mathrm{c}} / \mathrm{F}$ are estimated to be 14 and $24 \%$ lower in females compared with males, which results in $16 \%$ higher $\mathrm{AUC}$ and $26 \%$ higher $\mathrm{C}_{\max }$ estimates in females compared with males, a non-clinically relevant effect on upadacitinib exposures. It is worth noting that the estimate of the effect of sex on upadacitinib CL/F and $V_{\mathrm{c}} / \mathrm{F}$ was similar whether serum creatinine or creatinine clearance was included in the final model, indicating that the inclusion of creatinine clearance (which has a factor for sex in its calculation) does not underestimate the small effect 


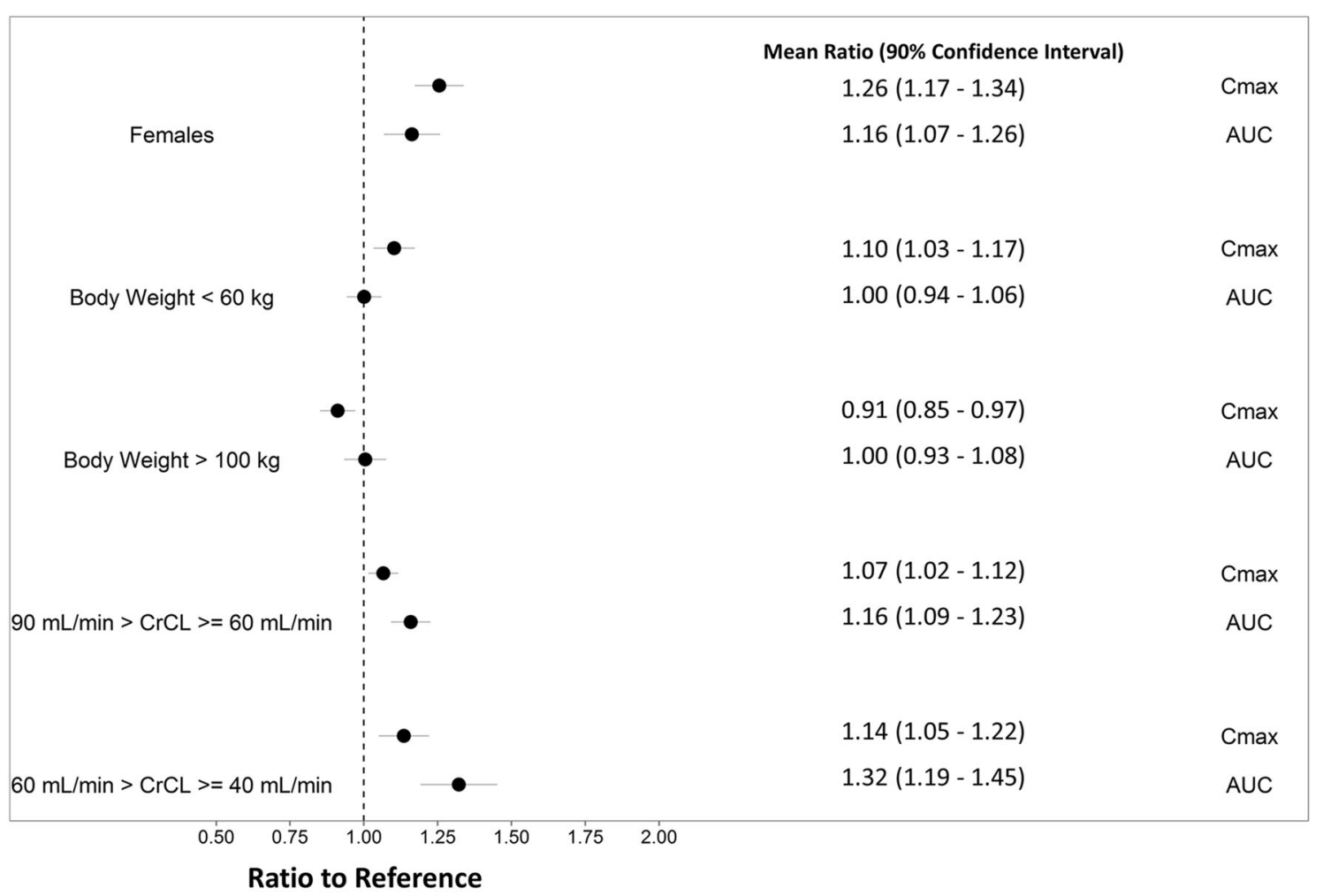

Fig. 4 Effect of covariates on upadacitinib exposures in rheumatoid arthritis patients (mean ratio and $90 \%$ confidence interval). Reference groups for the ratios presented: males (reference for females), body weight $\geq 60 \mathrm{~kg}$ and $\leq 100 \mathrm{~kg}$ (for body weight effect), and

of sex on upadacitinib pharmacokinetic parameters. Total body weight was statistically significantly correlated with $V_{\mathrm{c}} / \mathrm{F}$; however, this correlation is not expected to result in a clinically meaningful difference in exposures between subjects with different body weights (Fig. 4). Matched on sex and creatinine clearance, subjects with body weight $<60 \mathrm{~kg}$ or $>100 \mathrm{~kg}$ are predicted to have the same AUC and approximately $10 \%$ higher or lower $C_{\max }$, respectively, compared with subjects with body weight between 60 and $100 \mathrm{~kg}$. Overall, none of the covariates described above warrant adjustment of upadacitinib dose in specific patient subpopulations.

ISV in upadacitinib CL/F and $V_{\mathrm{c}} / \mathrm{F}$ was estimated to be 26 and $27 \%$, respectively, in subjects with RA, and 16 and $14 \%$, respectively, in healthy subjects, indicating modest variability in upadacitinib pharmacokinetics (Table 3). ISV for the $K_{a}$ was estimated to be relatively higher (150\%); this estimate is likely due to (1) the inclusion of phase II data with some samples collected in the absorption phase, and the expected less-than-optimal accuracy in recording the exact sampling time relative to dosing in a phase II setting; and (2) combining data from subjects who received upadacitinib under fed and fasting conditions in both
$\mathrm{CrCL} \geq 90 \mathrm{~mL} / \mathrm{min}$ (for $\mathrm{CrCL}$ effect). $C r C L$ creatinine clearance, $C_{\max }$ maximum concentration, $A U C$ area under the concentrationtime curve

phase I and II studies. Food was shown to affect upadacitinib absorption rate (food decreased upadacitinib $C_{\max }$ from the immediate-release formulation by approximately $20 \%$ and delayed time to $C_{\max }\left[T_{\max }\right]$ by approximately $2 \mathrm{~h}$ compared with the administration of upadacitinib under fasting conditions), with no impact on upadacitinib absorption extent (i.e. no impact of food on upadacitinib AUC) [23]. Given that this small effect of food on upadacitinib $C_{\max }$ and $T_{\max }$ is not clinically relevant, upadacitinib was administered in the phase II studies included in this analysis without regard to food, and information on the prandial status prior to dose administration in the studies was not captured. Therefore, the fasting/fed status was not evaluated in the model as a fixed effect on upadacitinib $K_{\mathrm{a}}$.

The population pharmacokinetic model described in this report was utilized in the characterization of the relationships between upadacitinib exposures and different efficacy endpoints in RA. The lack of clinical relevance of the covariates identified as statistically significant in the population pharmacokinetic analysis was further confirmed within the exposure-response analysis framework. Detailed reports of these exposure-response analyses are warranted. 
Furthermore, additional analyses of the ongoing phase III trials of upadacitinib in subjects with RA will further inform these conclusions.

In vitro, upadacitinib is metabolized by CYP3A (nonsensitive substrate), with a potentially minor metabolic contribution from CYP2D6. The strong CYP3A inhibitor ketoconazole has been shown to increase upadacitinib exposures by approximately 75\% [23]. Approximately 5\% of subjects included in the analysis dataset were reported to have used a CYP3A or CYP2D6 inhibitor concomitantly while receiving upadacitinib. No effect of concomitant use of CYP3A (moderate or weak) or CYP2D6 inhibitors could be detected on upadacitinib $\mathrm{CL} / \mathrm{F}$ in this population analysis; however, this assessment is limited by the small number of subjects in the analysis dataset who used these medications and the short treatment duration for several concomitant medications of interest. Approximately $60 \%$ of subjects included in the analysis had CYP2D6 extensive metabolizer phenotype (as classified based on the pharmacogenetic analysis). CYP2D6 phenotype was not a statistically significant covariate on upadacitinib clearance, which is in agreement with in vitro data showing only minor contribution of CYP2D6 to upadacitinib metabolism.

\section{Conclusions}

Upadacitinib pharmacokinetics were best described by a two-compartment model with first-order absorption and elimination. Upadacitinib pharmacokinetics did not significantly deviate from linearity over the dose range evaluated. Sex, renal impairment (mild and moderate), and body weight showed statistically significant, but not clinically relevant, effects on upadacitinib pharmacokinetics. Therefore, upadacitinib doses are being administered in the ongoing phase III trials in RA and PsA without regard for baseline characteristics. Additional analyses are warranted when data from large phase III studies become available.

\section{Compliance with Ethical Standards}

Funding The studies were sponsored by AbbVie. AbbVie contributed to the study design, research, and interpretation of data, and the writing, review, and approval of the publication. Medical writing support was provided by Therese Stickler under contract with AbbVie.

Conflict of interest Drs. Klünder, Mohamed, and Othman are employees and shareholders of AbbVie.

Ethical approval The studies were conducted in accordance with Good Clinical Practice guidelines and the ethical principles that have their origin in the Declaration of Helsinki. The protocols and informed consent forms were approved by the Institutional Review Board at each site.
Informed consent All participants provided written informed consent before any study-related procedures were performed.

Open Access This article is distributed under the terms of the Creative Commons Attribution-NonCommercial 4.0 International License (http://creativecommons.org/licenses/by-nc/4.0/), which permits any noncommercial use, duplication, adaptation, distribution and reproduction in any medium or format, as long as you give appropriate credit to the original author(s) and the source, a link is provided to the Creative Commons license and any changes made are indicated.

\section{References}

1. Firestein GS. Evolving concepts of rheumatoid arthritis. Nature. 2003;423(6937):356-61.

2. Wilkie WS, Schwieterman P. Strategies for the management of rheumatoid arthritis. Orthopedics. 2012;35(2):125-30.

3. Nakayamada S, Kubo S, Iwata S, Tanaka Y. Recent progress in JAK inhibitors for the treatment of rheumatoid arthritis. BioDrugs. 2016;30(5):407-19.

4. Norman P. Selective JAK inhibitors in development for rheumatoid arthritis. Expert Opin Investig Drugs. 2014;23(8):1067-77.

5. AbbVie. A study comparing ABT-494 to placebo and to adalimumab in subjects with rheumatoid arthritis who are on a stable dose of methotrexate and who have an inadequate response to methotrexate (SELECT-COMPARE). ClinicalTrials.gov identifier NCT02629159. https://clinicaltrials.gov/ct2/show/ NCT02629159. Accessed 25 Apr 2017.

6. AbbVie. A study comparing ABT-494 to placebo in subjects with rheumatoid arthritis on a stable dose of conventional synthetic disease-modifying antirheumatic drugs (csDMARDs) who have an inadequate response to csDMARDs alone (SELECT-NEXT). ClinicalTrials.gov identifier NCT02675426. https://clinicaltrials. gov/ct2/show/NCT02675426. Accessed 25 Apr 2017.

7. AbbVie. A study to compare ABT-494 to placebo in subjects with rheumatoid arthritis on stable dose of conventional synthetic disease-modifying antirheumatic drugs (csDMARDs) who have an inadequate response or intolerance to biologic DMARDs (SELECT-BEYOND). ClinicalTrials.gov identifier NCT02706847. https://clinicaltrials.gov/ct2/show/ NCT02706847. Accessed 25 Apr 2017.

8. AbbVie. A study to compare ABT-494 monotherapy to methotrexate monotherapy in subjects with rheumatoid arthritis (RA) who have not previously taken methotrexate (SELECTEARLY). ClinicalTrials.gov identifier NCT02706873. https:// clinicaltrials.gov/ct2/show/NCT02706873. Accessed 25 Apr 2017.

9. AbbVie. A study comparing ABT-494 monotherapy to methotrexate (MTX) monotherapy in subjects with rheumatoid arthritis (RA) who have an inadequate response to MTX (SELECT-MONOTHERAPY). ClinicalTrials.gov identifier NCT02706951. https://clinicaltrials.gov/ct2/show/ NCT02706951. Accessed 25 Apr 2017.

10. AbbVie. A phase 3 study to compare ABT-494 to abatacept in subjects with rheumatoid arthritis on stable dose of conventional synthetic disease-modifying antirheumatic drugs (csDMARDs) who have an inadequate response or intolerance to biologic DMARDs (SELECT-CHOICE). ClinicalTrials.gov Identifier NCT03086343. https://clinicaltrials.gov/ct2/show/ NCT03086343. Accessed 25 Apr 2017. 
11. AbbVie. A study comparing ABT-494 to placebo in participants with active psoriatic arthritis who have a history of inadequate response to at least one biologic disease modifying anti-rheumatic drug (SELECT-PsA 2). ClinicalTrials.gov identifier NCT03104374. https://clinicaltrials.gov/ct2/show/ NCT03104374. Accessed 25 Apr 2017.

12. AbbVie. A study comparing ABT-494 to placebo and to adalimumab in participants with psoriatic arthritis who have an inadequate response to at least one non-biologic disease modifying anti-rheumatic drug (SELECT-PsA 1). ClinicalTrials.gov identifier NCT03104400. https://clinicaltrials.gov/ct2/show/ NCT03104400. Accessed 25 Apr 2017.

13. AbbVie. A multicenter, randomized, double-blind, placebo-controlled study of ABT-494 for the induction of symptomatic and endoscopic remission in subjects with moderately to severely active Crohn's disease who have inadequately responded to or are intolerant to anti-TNF therapy (Celest Study). ClinicalTrials.gov identifier NCT02365649. https://clinicaltrials.gov/ct2/show/ NCT02365649. Accessed 25 Apr 2017.

14. AbbVie. A study to evaluate ABT-494 in adult subjects with moderate to severe atopic dermatitis. ClinicalTrials.gov identifier NCT02925117. NCT02925117. Accessed 25 Apr 2017.

15. AbbVie. A study to evaluate the safety and efficacy of ABT-494 for induction and maintenance therapy in subjects with moderately to severely active ulcerative colitis. ClinicalTrials.gov identifier NCT02819635. https://clinicaltrials.gov/ct2/show/ NCT02819635. Accessed 25 Apr 2017.

16. Mohamed MF, Camp HS, Jiang P, Padley RJ, Asatryan A, Othman AA. Pharmacokinetics, safety and tolerability of ABT-494, a Novel selective JAK 1 inhibitor, in healthy volunteers and subjects with rheumatoid arthritis. Clin Pharmacokinet. 2016;55(12):1547-58.

17. Kremer JM, Emery P, Camp HS, Friedman A, Wang L, Othman AA, et al. A phase IIb study of ABT-494, a selective JAK-1 inhibitor, in patients with rheumatoid arthritis and an inadequate response to anti-tumor necrosis factor therapy. Arthritis Rheumatol. 2016;68(12):2867-77.
18. Genovese MC, Smolen JS, Weinblatt ME, Burmester GR, Meerwein S, Camp HS, et al. Efficacy and safety of ABT-494, a Selective JAK-1 inhibitor, in a phase IIb study in patients with rheumatoid arthritis and an inadequate response to methotrexate. Arthritis Rheumatol. 2016;68(12):2857-66.

19. Sheiner LB, Ludden TM. Population pharmacokinetics/dynamics. Annu Rev Pharmacol Toxicol. 1992;32:185-209.

20. Beal SL. Ways to fit a PK model with some data below the quantification limit. $\mathrm{J}$ Pharmacokinet Pharmacodyn. 2001;28(5):481-504.

21. Bergstrand M, Hooker AC, Wallin JE, Karlsson MO. Predictioncorrected visual predictive checks for diagnosing nonlinear mixed-effects models. AAPS J. 2011;13(2):143-51.

22. Petersson KJ, Hanze E, Savic RM, Karlsson MO. Semiparametric distributions with estimated shape parameters. Pharm Res. 2009;26(9):2174-85.

23. Mohamed MF, Jungerwirth S, Asatryan A, Jiang P, Othman AA. Assessment of effect of CYP3A Inhibition, CYP Induction, OATP1B Inhibition, and high-fat meal on pharmacokinetics of the JAK1 inhibitor upadacitinib. $\mathrm{Br} \mathrm{J}$ Clin Pharmacol. 2017;83(10):2242-8.

24. US FDA. Clinical pharmacology and biopharmaceutics review(s) - tofacitinib. Silver Spring: Center for Drug Evaluation and Research; 2011.

25. Namour F, Diderichsen PM, Cox E, Vayssiere B, Van der Aa A, Tasset C, et al. Pharmacokinetics and pharmacokinetic/pharmacodynamic modeling of filgotinib (GLPG0634), a selective JAK1 Inhibitor, in support of phase IIB dose selection. Clin Pharmacokinet. 2015;54(8):859-74.

26. Dickmann LJ, Patel SK, Rock DA, Wienkers LC, Slatter JG. Effects of interleukin-6 (IL-6) and an anti-IL-6 monoclonal antibody on drug-metabolizing enzymes in human hepatocyte culture. Drug Metab Dispos. 2011;39(8):1415-22.

27. Xu Y, Hijazi Y, Wolf A, Wu B, Sun YN, Zhu M. Physiologically based pharmacokinetic model to assess the influence of blinatumomab-mediated cytokine elevations on cytochrome P450 enzyme activity. CPT Pharmacomet Syst Pharmacol. 2015;4(9):507-15. 